EPJ Web of Conferences 81, 06009 (2014)

DOI: 10.1051/epjconf/ 20148106009

(C) Owned by the authors, published by EDP Sciences, 2014

\title{
Electromagnetic Calorimeter for HADES Experiment
}

P. Rodríguez-Ramos ${ }^{1,10, a}$, L. Chlad ${ }^{1,11}$, E. Epple ${ }^{4}$, L. Fabbietti ${ }^{4}$, T. Galatyuk ${ }^{5}$, M. Golubeva ${ }^{6}$, F. Guber ${ }^{6}$, S. Hlaváč ${ }^{7}$, A. Ivashkin ${ }^{6}$, M. Kajetanowic ${ }^{8}$, B. Kardan ${ }^{2}$, W. Koenig ${ }^{9}$, G. Korcyl ${ }^{8}$, A.

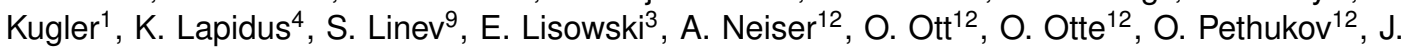

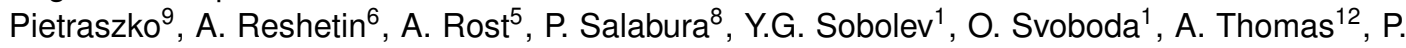
Tlusty ${ }^{1}$ and M. Traxler ${ }^{9}$

for the HADES Collaboration

${ }^{1}$ Nuclear Physics Institute, Academy of Sciences of the Czech Republic, Řež, Czech Republic

${ }^{2}$ Institut für Kernphysik, Goethe-Universität Frankfurt, Germany

${ }^{3}$ Institute of Technology Cracow, Cracow, Poland

${ }^{4}$ Excellence Cluster "Origin and Structure of the Universe" Technische Universität München, Garching, Germany

${ }^{5}$ Technische Universität Darmstadt, Darmstadt, Germany

${ }^{6}$ Institute for Nuclear Research, Russian Academy of Sciences, Moscow, Russia

${ }^{7}$ Institute of Physics, Slovak Academy of Sciences, Bratislava, Slovakia

${ }^{8}$ Smoluchowski Institute of Physics, Jagiellonian University of Krakow, Cracow, Poland

${ }^{9} \mathrm{GSI}$ Helmholtzzentrum für Schwerionenforschung GmbH, Darmstadt, Germany

${ }^{10}$ Czech Technical University in Prague, Faculty of Nuclear Sciences and Physical Engineering, Prague,

Czech Republic

${ }^{11}$ Faculty of Mathematics and Physics, Charles University in Prague, Prague, Czech Republic

${ }^{12}$ Institut für Kernphysik, Johannes Gutenberg-Universität, Mainz, Germany

\begin{abstract}
Electromagnetic calorimeter (ECAL) is being developed to complement dilepton spectrometer HADES. ECAL will enable the HADES@FAIR experiment to measure data on neutral meson production in heavy ion collisions at the energy range of 2-10 AGeV on the beam of future accelerator SIS100@FAIR. We will report results of the last beam test with quasi-monoenergetic photons carried out in MAMI facility at Johannes Gutenberg Universität Mainz.
\end{abstract}

\section{Introduction}

The electromagnetic calorimeter (ECAL) will extend the HADES experimental possibilities in several directions. First of all, the ECAL will enable measurement of photons from various sources like meson decays or decays of neutral $\Lambda(1405)$ and $\Sigma(1385)$ resonances. The ECAL will also improve electron/pion separation at large momenta over $400 \mathrm{MeV} / \mathrm{c}$. Basic design of the ECAL is given by the HADES geometry - six separate sectors covering almost full azimuthal angle and polar angle $18^{\circ}$ to $45^{\circ}$.

\footnotetext{
a e-mail: ramos@ujf.cas.cz
} 


\section{Experimental layout}

A new beam test of calorimeter modules and read-out concepts was performed between $7^{\text {th }}$ and $10^{\text {th }}$ January 2014. Line beam with energy of $1508.4 \mathrm{MeV}$ was directed to a copper target, energy of produced photons was determined using the method of tagged electrons in dedicated TAGGER [? ] detector in MAMI A2 hall. Four ECAL [? ] modules were placed on a movable platform one meter behind the tagger shielding. Photon beam cross section was of $6 \mathrm{~mm}$ diameter. One ECAL module was equipped with 1.5" inch PMT EMI 9903KB. Two modules were equipped with 3" Hamamatsu R6091 PMT's and the last module contained 1" Hamamatsu R8619 PMT.

\subsection{Relative energy resolution}

The energy resolution of the ECAL modules equipped with 1", 1.5" and 3" PMTs deliver comparable relative energy resolution (5.8\% at $1 \mathrm{GeV}$ photon with 1.5 " EMI and 5.5\% with 3" Hamamatsu), whereas the module with 1" Hamamatsu PMT gives by $1.6 \%$ worse energy resolution and non-linear response.

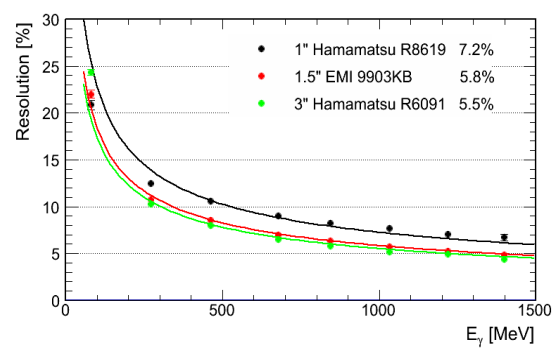

Figure 1. Relative energy resolution of calorimeter modules equipped with different photomultipliers.

\subsection{Energy leakage into neighbor module parallel with the beam}

Two identical modules equipped with 3" PMT were irradiated with photon beam parallel with the module $Z$ axis (along the module). Three measurements were done with the beam position in the center of the first module, $1 \mathrm{~cm}, 2 \mathrm{~cm}, 3 \mathrm{~cm}$ and $4 \mathrm{~cm}$ from the center closer to the second module. Most of the energy was still deposited in the first module in the case of beam in the center or two centimeters far from the center of the module and it was possible to fully recover original photon. Unfortunately some energy is lost in the case of photon beam placed close to the module border, most probably due to brass walls between the modules. Some of the secondary particles (mainly electrons) created in the gamma interaction interact with the non-active volume of the detector and are lost, see Fig. 1. 

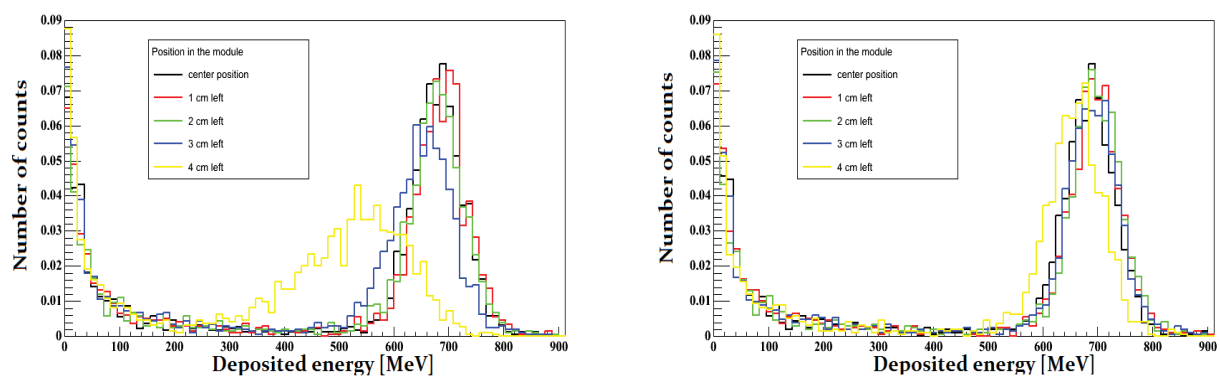

Figure 2. Energy deposited in the first module hit by the beam (left) and sum of energies deposited in both neighboring modules (right). Two cases for photon beam energy $680 \mathrm{MeV}$.

\subsection{Energy leakage into neighbor module inclined $6^{\circ}$ and $12^{\circ}$ respect to the longitudinal axis}

The response of the ECAL modules on photons coming under non-zero angle is very important as most of the modules will be declined with respect to the incoming particles. Two sets of measurements with photon inclination angle $6^{\circ}$ and $12^{\circ}$ were done. Beam was moved over the front surface of one module in the steps $-2 \mathrm{~cm}, 0 \mathrm{~cm}$ (center of the module), $+2 \mathrm{~cm}$ closer to the second module, and $+4 \mathrm{~cm}$. The original photon energy was recovered as a sum of energies stored in single modules. It was demonstrated, that almost no energy is lost between the modules and we are able to recover original energy of photon, see Fig. 2.
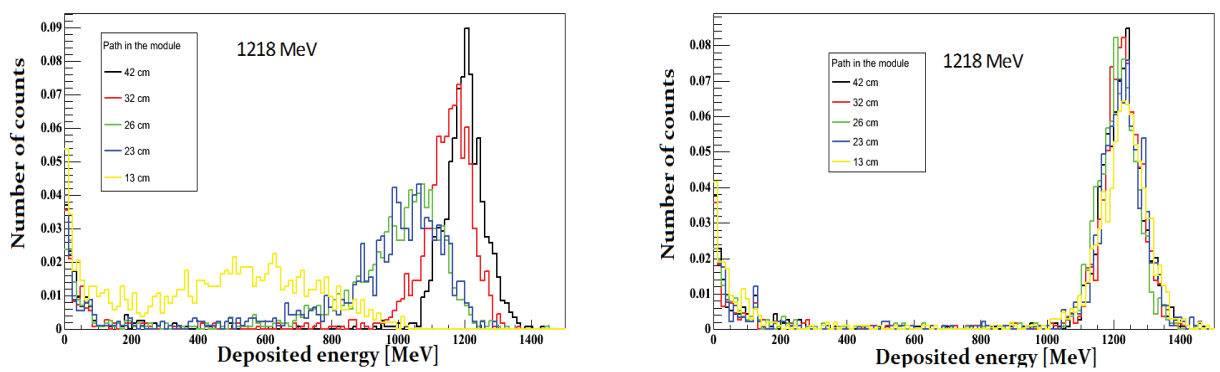

Figure 3. Energy deposited in one module hit by the photon beam (left) and sum of energies deposited in both neighboring modules (right). Two cases for photon beam energy $1217.8 \mathrm{MeV}$.

\section{Conclusions}

Both 1.5" and 3" PMTs fulfil the requirements for the required resolution. Energy resolution was studied to decide the size of PMT taking into account physical and economic point of view. Energy leakage between the neighbor modules was tested with parallel and declined beams. Energy of original photon was successfully recovered as a sum of energies deposited in each of the modules. Some 
part of the original energy was lost only in the case of parallel photon beam placed close to module boarder. This effect can be corrected for using results of the presented test.

\section{Acknowledgements}

We would like to express special thanks to the staff of the MAMI facility for providing excellent beam conditions. Work supported by GSI, HIC for FAIR, EMMI and Czech MSMT LG 120007, GACR 13-067595 and AS CR M100481202 grants. This work has been supported by the European Community FP7 - Capacities, contract HadronPhysics3 n² 283286"

\section{References}

[1] J. C. McGeorge, J. D. Kellie, J. R. M. Annand et al. Upgrade of the Glasgow photon tagging spectrometer for Mainz MAMI-C. 37 (2008) 129.

[2] O. Svoboda et al. (HADES Collaboration), Electromagnetic calorimeter for the HADES@FAIR experiment, 9 C05002, (2014) 